\title{
Interdigitation in spin-coated lipid layers in air
}

DOI:

10.1016/j.colsurfb.2018.08.041

\section{Document Version}

Accepted author manuscript

Link to publication record in Manchester Research Explorer

\section{Citation for published version (APA):}

Dols-perez, A., Fumagalli, L., \& Gomila, G. (2018). Interdigitation in spin-coated lipid layers in air. Colloids and Surfaces B: Biointerfaces, 172, 400-406. https://doi.org/10.1016/j.colsurfb.2018.08.041

\section{Published in:}

Colloids and Surfaces B: Biointerfaces

\section{Citing this paper}

Please note that where the full-text provided on Manchester Research Explorer is the Author Accepted Manuscript or Proof version this may differ from the final Published version. If citing, it is advised that you check and use the publisher's definitive version.

\section{General rights}

Copyright and moral rights for the publications made accessible in the Research Explorer are retained by the authors and/or other copyright owners and it is a condition of accessing publications that users recognise and abide by the legal requirements associated with these rights.

\section{Takedown policy}

If you believe that this document breaches copyright please refer to the University of Manchester's Takedown Procedures [http://man.ac.uk/04Y6Bo] or contact uml.scholarlycommunications@manchester.ac.uk providing relevant details, so we can investigate your claim.

\section{open 2 Access}




\title{
Interdigitation in spin-coated lipid layers in air
}

\author{
Aurora Dols-Perez ${ }^{+*}$, Laura Fumagalli ${ }^{\ddagger}$ and Gabriel Gomila ${ }^{l l, \neq}$
}

\author{
${ }^{\dagger}$ Department of Bionanoscience, Kavli Institute of Nanoscience, Delft University of Technology, Van der \\ Maasweg 9, 2629 HZ Delft, Netherlands \\ ${ }^{\ddagger}$ School of Physics and Astronomy, University of Manchester, Oxford Road, Manchester M13 9PL, \\ United Kingdom \\ "Institut de Bioenginyeria de Catalunya (IBEC), C/ Baldiri i Reixac 15-21, 08028 Barcelona, Spain \\ ${ }^{\ddagger}$ Departament d'Electrònica, Universitat de Barcelona, C/ Martí i Franquès 1, 08028 Barcelona, Spain

\section{Corresponding Author} \\ *corresponding author: Aurora Dols-Perez (a.dolsperez@tudelft.nl)

\section{ABSTRACT}

In this study, we show that dry saturated phospholipid layers prepared by the spin-coating technique could present thinner regions associated to interdigitated phases under some conditions. The morphological characteristics of lipid layers of saturated phosphocholines, such as dilauroylphosphatidylcholine (DLPC), dimyristoylphosphatidylcholine

(DMPC), dipalmitoylphosphatidylcholine (DPPC) and distearoylphosphatidylcholine (DSPC), have been measured by Atomic Force Microscopy and revealed that the presence of interdigitated regions is not induced by the same parameters that induce them in hydrated samples. To achieve these results the effect of the lipid hidrocabonated chain length, the presence of alcohol in the coating solution, the spinning velocity and the presence of cholesterol were tested. We showed that DPPC and DSPC bilayers, on the one side, can show structures with similar height than interdigitated regions observed in hydrated samples, while, on the other side, DLPC and DMPC tend to show no evidence of interdigitation. Results indicate that the presence of interdigitated areas is due to the presence of lateral tensions and, hence, that they can be eliminated by releasing these tensions by, for instance, the addition of cholesterol. These results demonstrate that interdigitation in lipid layers is a rather general phenomena and can be observed in lipid bilayers in dry conditions.

KEYWORDS : Spin-coating, lipid layers, Atomic Force Microscopy, Interdigitation 


\section{Introduction}

Model lipid membranes have been widely used to facilitate the comprehension of natural membranes properties by reducing complexity and simplifying their preparation [1-7]. They allow, under experimentally well controlled conditions, the reconstruction of membrane structures, the study of the interactions between specific membrane components and also the effect of other elements (e.g. ions, proteins, drugs, etc.).

Apart from these fundamentals studies, model lipid membranes have also been used as platforms for other applications, such as lipid-assisted assays and biosensors [8-11]. In these applications, the resistance and morphological stability in dried or low humidity environments is an important feature. But, at present, relatively little information is available on the nanostructure of dry lipid bilayers, as most of the studies performed so far have focused on the production and analysis of hydrated lipid bilayers.

In recent years, the use of the spin-coating technique has allowed obtaining high-quality and morphologically stable dry lipid layers in air [12-17] on different supports [18-20]. This advancement opened the possibility to address its nanoscale and nanomechanical properties in a reliable and relatively simple way. Dry and stable single component lipid layers of DOPC and SM have been produced as well as binary and ternary mixtures containing cholesterol, including lipid raft models such as DOPC/ SM/ Chol $[21,22]$. The study of these systems showed the presence of liquid disordered, liquid ordered and gel phases in dry lipid samples, with morphological properties (e.g. layer thicknesses) in similarity to those observed on the same systems under hydrated conditions and prepared by different techniques such as, the vesicle fusion [23], Langmuir-Blodgett/Schäfer [24], microcontact printing [25] or lipid dippen nanolithography[26].

In the present study we investigate a subtler phenomena, i.e. the presence/absence of thinner regions associated to interdigitated phase $\left(L_{\beta} \mid\right)$ in saturated phosphocholines in dry conditions. Interdigitated phases have been widely observed on hydrated lipid bilayers. This phase consists of a thinning of the bilayer height, which could be partial or of the total area [27, 28], due to the interpenetration and disorder or tilting of the acyl chains and tilt of their angle [27, 29-33]. Derived from that there is also an expansion of the bilayer area laterally [29, 34, 35]. This phase is produced in phosphatidylcholines in solution due the presence of alcohols $[27,28,32,34,35]$, volatile anesthetics [36, 37] or by the presence of an abnormal pressure, such as, the interaction with the substrate or the presence of lateral tensions 
$[28,38,39]$. Moreover, it is known that Cholesterol and other sterols play a role in the interdigitating process enhancing or reducing the effect of ethanol depending on the sterol content [38, 40-42].

The existence of an interdigitated phase in dry lipid layers has not been reported to date. In this study, we precisely show that dry saturated phospholipid layers prepared by the spin-coating technique can present thinner regions associated to interdigitated phase in some conditions. Atomic Force Microscopy (AFM) offers the appropriate lateral and vertical resolution to characterize lipid bilayers in different media, air or liquid, and to distinguish heights corresponding to different lipid phases [38, 43, 44]. In fact the value of these characteristic heights, has been used extensively in literature to estimate the phase present in a sample and also the presence of interdigitated regions[28, 35]. In this study, we investigated by AFM the morphology of lipid layers prepared by the spin-coating technique in dry air and the influence of different parameters in the presence of these thinner regions, such as, the phospholipid chain length, and consequently of different transition temperatures $T_{m}$, the presence of alcohols in the coating solution, the spinning velocity and the cholesterol concentration.

\section{Experimental section}

2.1. Materials: Lipid layers were prepared with 1,2-dilauroyl-sn-glycero-3-phosphocholine (DLPC), 1,2dimyristoyl-sn-glycero-3-phosphocholine (DMPC), 1,2-dipalmitoyl-sn-glycero-3-phosphocholine (DPPC) and 1,2-distearoyl-sn-glycero-3-phosphocholine (DSPC) purchased from Sigma-Aldrich and used as received without further purification. Hexane, LC-MS grade (Sigma-Aldrich), Isopropanol (Sigma-Aldrich) and Methanol, HPLC grade (Sigma-Aldrich), were used as solvent in the experiments. Hi-grade freshly cleaved mica substrates (Ted Pella,Inc) were used as support.

2.2. Sample preparation and AFM imaging: Air-stable lipid layers have been obtained by the spincoating technique following the methodology developed by Simonsen and Bagatolli [12] further optimized to produce ultrathin (single) bilayer samples [21]. In here, the concentration of lipid used in the coating solution is $1 \mathrm{mM}$. Briefly, a small volume of lipid stock solution was deposited on the highgrade freshly cleaved mica and spun with a spinner (WS-650MZ-23NPP/LITE, Laurell Technologies Corp.) for $1 \mathrm{~min}$ immediately after the deposition of the solution. The speed used was 3000rpm unless is directly specified in the text. Next, the samples were placed under vacuum in a desiccator during 15-20 
$1 \mathrm{~h}$ to fully evaporate the solvents. Fresh lipid solutions were prepared on the day of each experiment to avoid solvent evaporation and change in the lipid concentration.

AFM imaging was performed at $25^{\circ} \mathrm{C}$ in dry environment in a closed chamber with $\mathrm{N}_{2}$ stream and relative humidity $\mathrm{RH} \sim 0 \%$. Calibrated AFM probes (PPP-CONTR, Nanosensors, nominal spring constant $0.2 \mathrm{~N} / \mathrm{m}$, tip radius $<7 \mathrm{~nm}$ ) and a commercial AFM (Nanotec Electronica S.L) were used. Images were processed by using the WSxM software [45]. Height analysis was performed using histogram analysis of the pixels over image areas of $1000 \mathrm{~nm} \times 1000 \mathrm{~nm}(\mathrm{~N}=3-7)$. The small size of the areas is selected to avoid systematic errors in height determination associated with image flattening. The obtained value is the mean of the values extracted at different areas and the error corresponds to the standard deviation (SD) of the means. By using the spin-coating technique it is possible to form incomplete layers that allow measuring directly the bilayer thickness. Bare mica was demonstrated to be not observable in samples prepared by this method at these lipid concentrations, since the bottom layer consists of a uniform lipid monolayer, as we showed earlier [21]. This fact does not affect the determination of the bilayer heights.

\section{Results and discussion}

\subsection{Presence of interdigitated phases in dry lipid bilayers as a function of acyl chain length}

Different saturated phosphocholines, with different chain length (12:0, 14:0, 16:0 and 18:0) were selected to evaluate their behavior in dry conditions. In Figures 1A-1D we show AFM topography images of the dilauroylphosphatidylcholine DLPC (12:0), dimyristoylphosphatidylcholine DMPC (14:0), dipalmitoylphosphatidylcholine DPPC (16:0) and distearoylphosphatidylcholine DSPC (18:0) samples, respectively. Using $1 \mathrm{mM}$ of lipid in the stock solution, the spin-coated sample of lipids is composed by an homogeneous monolayer background with a single inverted bilayer on top of which bilayer patches or rims, and occasionally some multilayer patches, form [21]. At higher concentrations the number of layers increases $[12,16,21,22]$. DLPC sample presented a single bilayer on the homogenous monolayer background while DMPC, DPPC and DSPC samples presented additional patches of a second bilayer (Fig.1). Apart from the surface-covering dependence, described previously [21, 22, 46], the most relevant feature of these images is the existence of some characteristic heights. In particular, the histogram analysis reveals the presence of a single characteristic height equal to $4.5 \pm 0.5 \mathrm{~nm}$ for DLPC (Fig. 1A) and to $4.9 \pm 0.5 \mathrm{~nm}$ for DMPC (Fig 1B), independently of the number of bilayers. These values 
1 are in agreement with single bilayer thickness measurements performed on the samples with the same 2 composition under hydrated conditions even with different preparation method (vesicle fusion) [47, 48].

3 Instead, Figs. 1C and 1D corresponding to the DPPC and DSPC samples, respectively, exhibit bilayers with 4 two characteristic heights in each of them. The lower heights were $3.7 \pm 0.4 \mathrm{~nm}$ and $3.8 \pm 0.6 \mathrm{~nm}$, while 5 the thicker ones were $5.6 \pm 0.5 \mathrm{~nm}$ and $6.3 \pm 0.7 \mathrm{~nm}$ (Fig.1F), for DPPC and DSPC, respectively. The later 6 values are in agreement, again, with the bilayer thickness values reported for DPPC and DSPC hydrated 7 samples from vesicle fusion [28, 43, 49, 50]. They also agree with the well-known linear relationship 8 between bilayer thickness and acyl chain length [50] (dashed line in Fig.1-F). Concerning the lower 9 height values observed in these samples, their values are higher than that of a monolayer, thus allowing 10 discarding the presence of partial delamination. These values perfectly match values reported in the 11 literature for interdigitated phases in hydrated supported lipid bilayers [28] (see Fig. 2). This indicate 12 that interdigitation could be also present in phospholipid bilayers in dry conditions. 

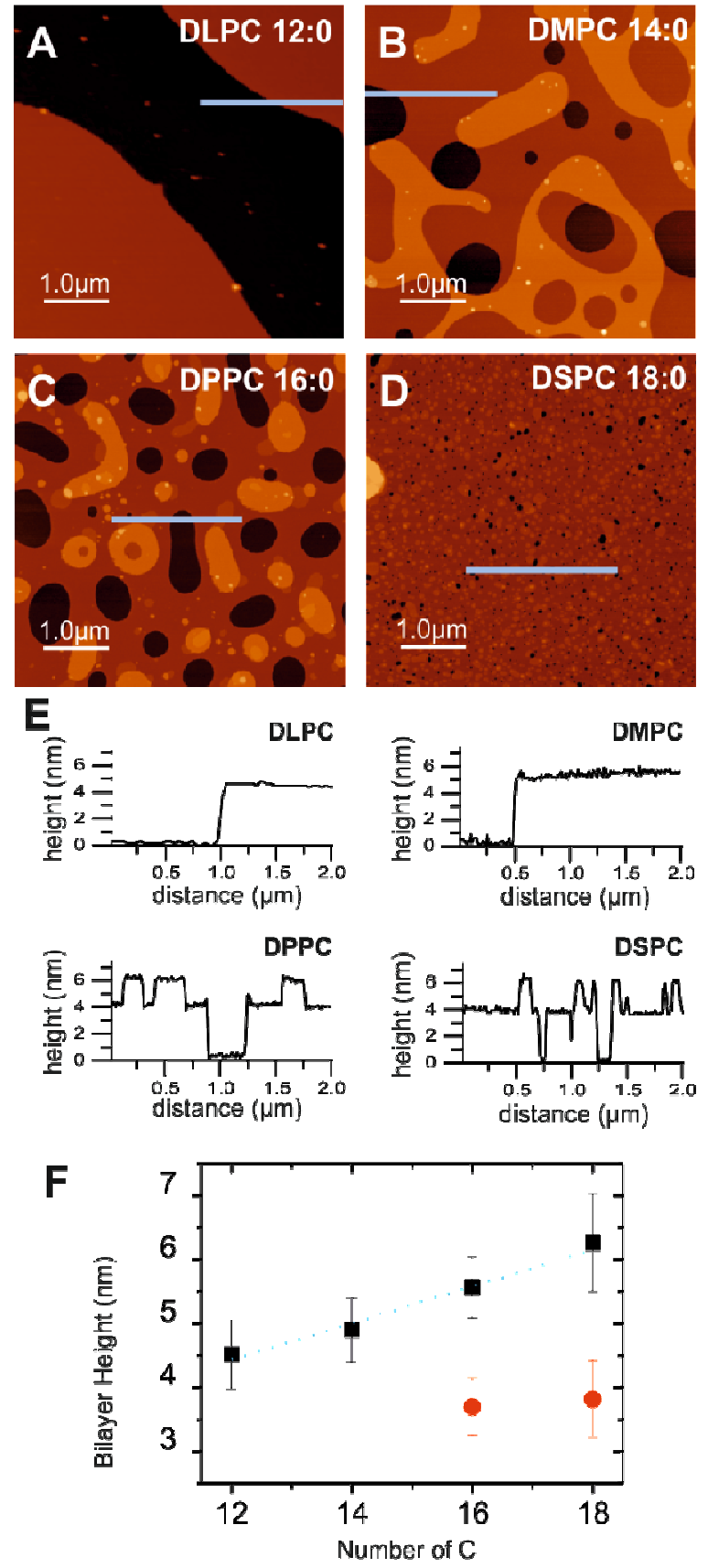

2 Figure 1. A-D) AFM topography image of spin-coated samples of DLPC (A), DMPC (B), DPPC (C) and DSPC 3 (D). Z-scale $=30 \mathrm{~nm}$. Figure E) Height profile of images A-D. Figure F) Plot representation of the bilayer 4 height extracted from the topography images vs number of $C$ of the acyl chain of the lipids used: DLPC 5 (12C), DMPC (14), DPPC (16) and DSPC (18). Black squares= higher regions, red dots= lower regions, 6 dashed line= bilayer height tendency as a function of the number of $C$. 


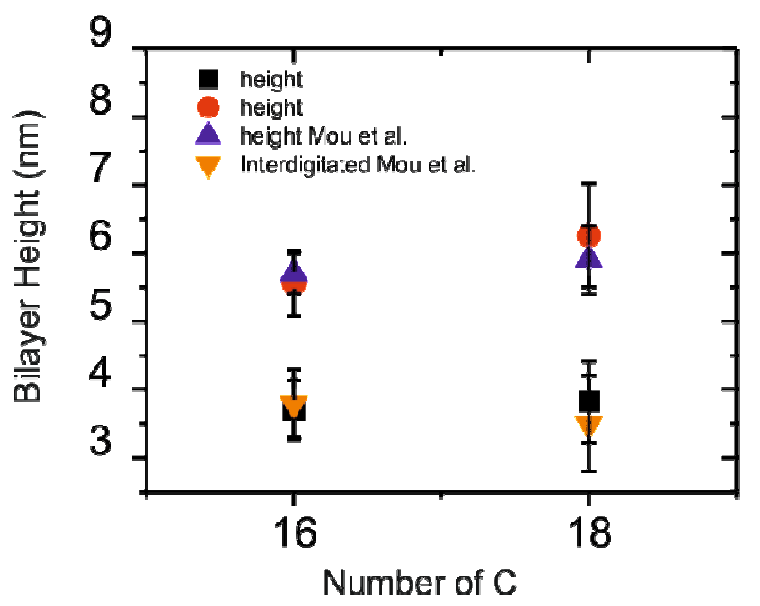

\subsection{Effect of the presence of alcohol in the coating solutions in the interdigitated phases}

Alcohols trigger the interdigitation in hydrated samples and, although a threshold value exists, the proportion of alcohol used is linked to the amount of area interdigitated and may reach the total surface of the bilayer for higher concentrations [28]. Alcohols increase the inter-headgroup distance by the orientation of their hydroxyl near the lipid headgroups and, from a certain concentration, the increment of the headgroup distance causes the lipid tails interdigitation [31, 51]. Furthermore, isopropanol is known to be more effective in the inducement of interdigitation than other alcohols, such as ethanol [28]. These effects have been studied when these alcohols are in the liquid solution in contact with the hydrated lipid bilayers.

In spin-coated samples in air, the alcohol or solvent is not incorporated to the membrane already prepared. It is used for the preparation of the lipid samples to dilute the lipids. This solvent should wet the substrate surface in which the spin-coating sample is formed and dissolve the lipids [12]. Due to the difficulty to dissolve saturated phospholipids in hydrocarbonated solvents such as hexane, the presence of alcohols in the coating solutions is necessary. Simonsen and coworkers proposed $\sim 3 \%$ of methanol in hexane to dissolve correctly these lipids [12] and other reported mixture to prepare these layers [52]. To check the effect of alcohols in the coating solution on the presence of the interdigitated structures, in other words if alcohols in solvent are responsible of the interdigitation, we have used mixtures of 
solvents and pure solvents (100\%). The solutions used were the previously mentioned hexane: methanol (98:2) mixture (referred to as solution A), pure methanol (solution B), pure isopropanol (solution C) and the solvent mixture isopropanol: hexane: water (3:1:1) (solution D). In the preparation of all samples the same speed $(3000 \mathrm{rpm})$ and spinning time $(1 \mathrm{~min})$ were used. AFM images of the lipid layers obtained with solvents $B, C$ and $D$ are given in Fig. 3, while results obtained with solvent $A$ are shown in Figure 1 .
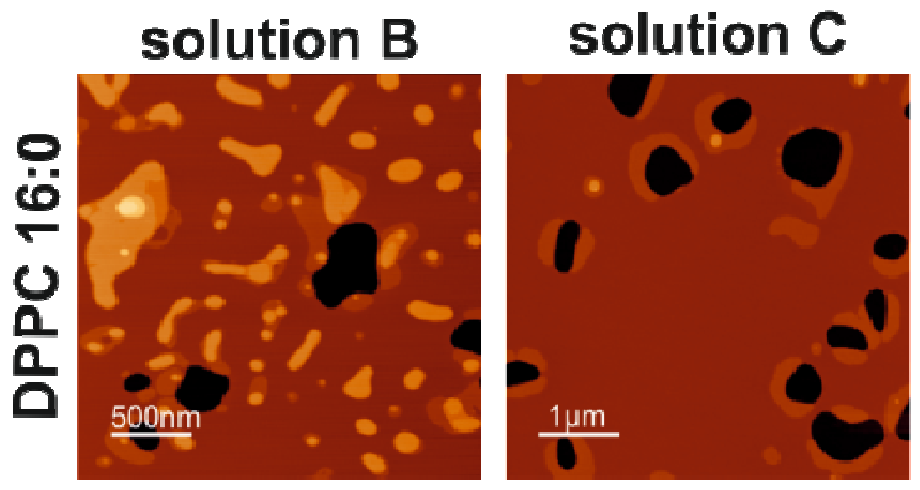

solution D
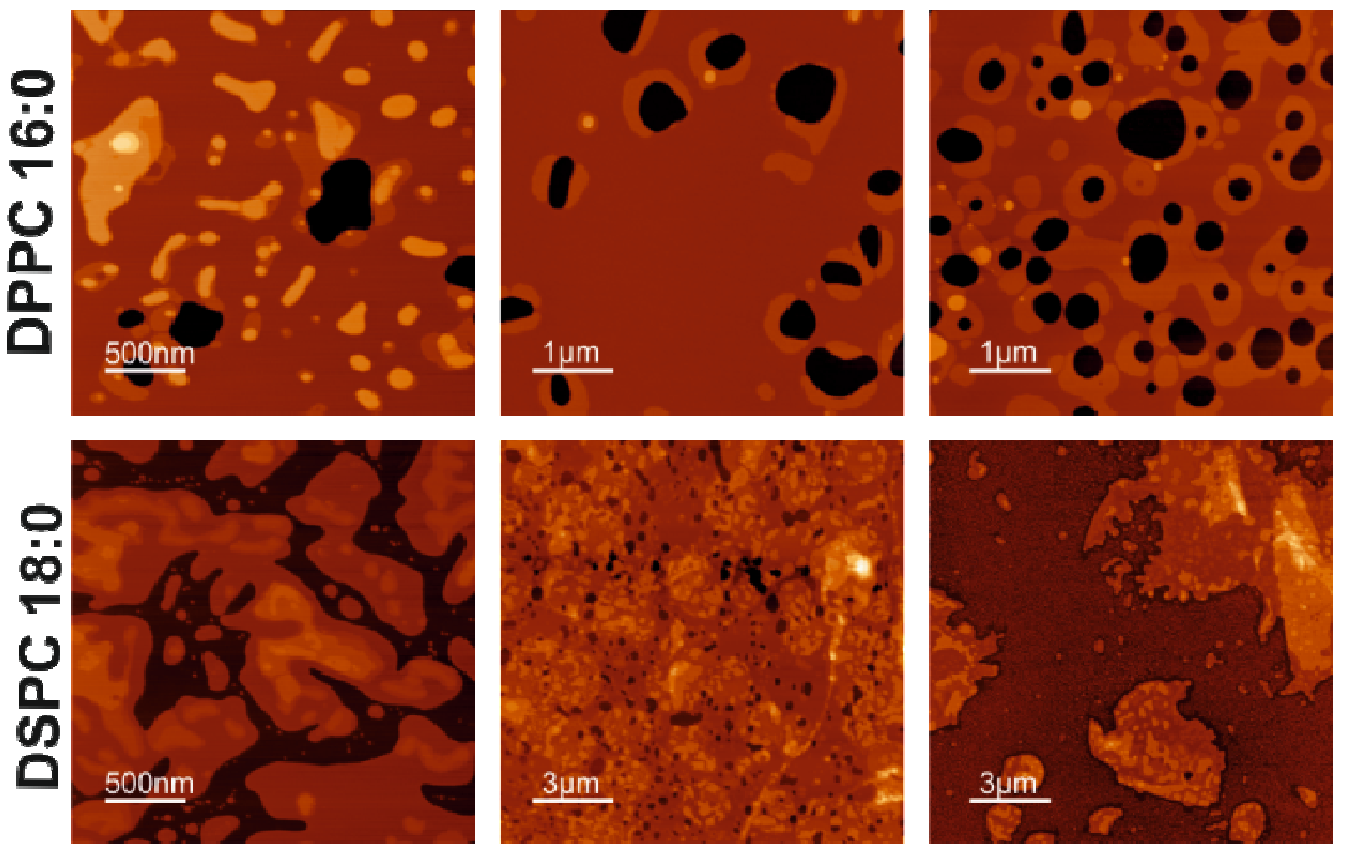

Figure 3. Representative AFM topography images of spin-coated samples of DPPC and DSPC (from top to bottom) in air for different solvents used (solvent B, solvent $C$ and solvent $D$ from left to right). Zscale DPPC images $=25 \mathrm{~nm}, \mathrm{z}$-scale DSPC images $=50 \mathrm{~nm}$.

Lower regions or thinning were not observed in DLPC and DMPC samples independently of the solvent used (data not shown). These results are in contrast with what observed on hydrated samples, where for instance alcohols produced the thinning of DMPC [53].

Figure 3 shows that the appearance of the DPPC and DSPC layers changes depending on the solvent used. But at the same time the presence of thinner regions (the interdigitated areas) is observed independently of the coating solution used and of the total amount of alcohol. No dependence of the interdigitated area with the solvent used was found. Results showed that the characteristic thickness of 
the bilayers is also maintained independently of the solvent used in both areas - interdigitated and noninterdigitated.

Moreover, no dependency of the extension of the affected area was observed in our results. Solutions with $100 \%$ of alcohols in the coating solution did not show a higher or complete interdigitation, contrary to observed in hydrated samples when the alcohol is incorporated after bilayer formation.

These results suggest that the presence of interdigitated regions in spin-coated samples of DPPC and DSPC was not induced by the presence of alcohols in the coating solutions.

\subsection{Effect of the lateral tension (spinning parameters) in the presence of interdigitated regions}

In hydrated samples the interdigitated phase induced by lateral tension $\left(L_{\beta}{ }^{\prime} I_{T}\right)$ is produced when the sample is exposed to a temperature above $\mathrm{Tm}$ and rapidly cooled below $\mathrm{Tm}$. The interdigitation is then produced due to the action of two factors: the substrate surface-lipid interaction and the tension created by having to pass from covering a large area to cover a smaller one in a short period of time [38, 39]. If the transition is not slow enough, the covered surface is larger than the expected for a solid phase and the bilayer cannot maintain its structure. For this reason, interdigitation over larger areas, requiring a higher area per molecule, is obtained.

During the spin-coating procedure the temperature was not changed. Therefore, another parameter should be responsible for changing the lateral tension of the sample. A clear candidate is the rotation speed or the acceleration of the spin-coating process. This hypothesis is compatible with the absence of interdigitated regions in DLPC and DMPC. These samples are in fluid phase the working temperature and their lateral mobility is higher than DPPC and DSPC samples. Hence, they can recover their configuration independently of the effect of the lateral pressure.

To test the possible effect of the rotation speed on the presence and structure of the interdigitated regions, we have prepared DPPC samples at various rotation speeds. In Fig. 4 we show the results obtained for samples prepared from $200 \mathrm{rpm}$ up to $5000 \mathrm{rpm}$. 

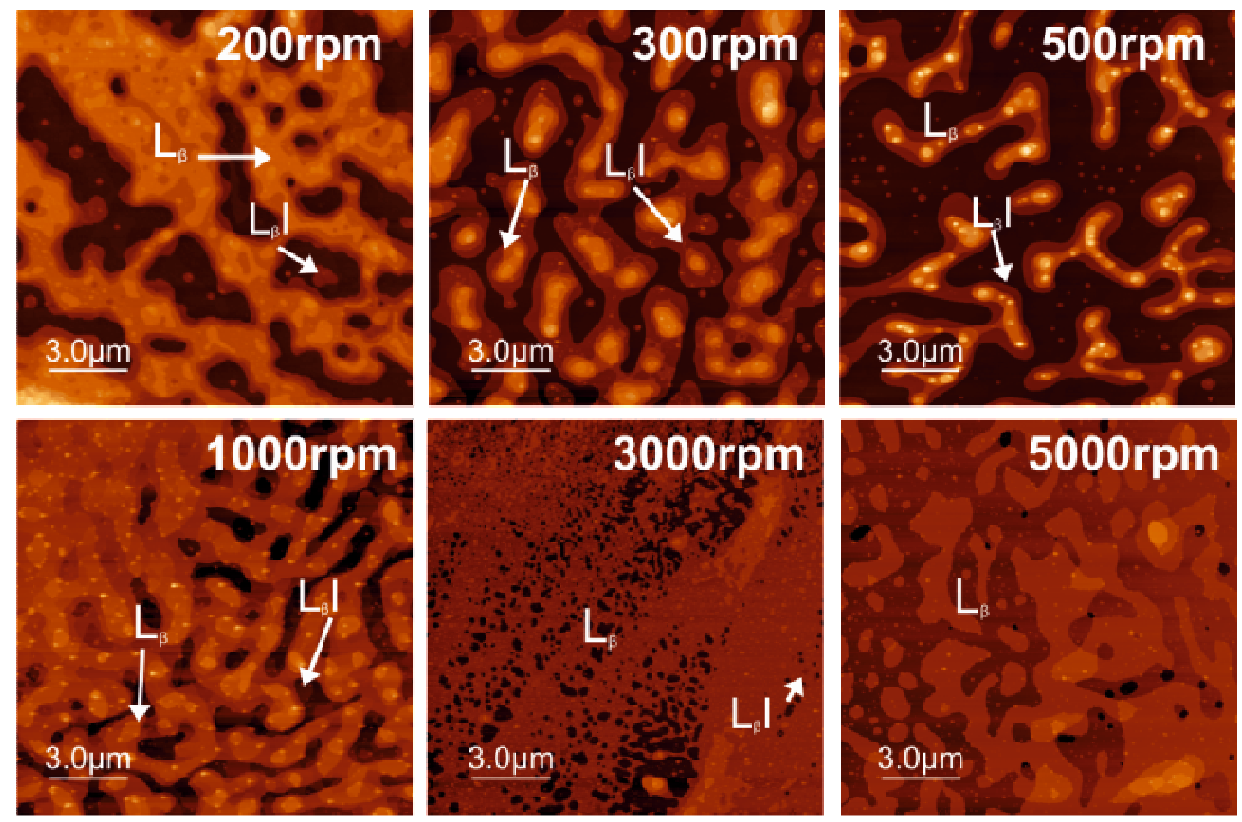

Figure 4. Representative AFM topography images of DPPC samples as a function of the rotation speed used during the sample preparation. Z-scale $=75 \mathrm{~nm}$.

The samples prepared at $200 \mathrm{rpm}, 300 \mathrm{rpm}$ and $500 \mathrm{rpm}$ showed large areas of background monolayer uncovered and also stacks of bilayers with heights corresponding to $L_{\beta} l$ and $L_{\beta}$. For higher rotation speeds $(1000 \mathrm{rpm}$ and $3000 \mathrm{rpm})$ the fraction of exposed monolayer became smaller, the height of the bilayers stacks is also reduced but the presence of the interdigitated phase was still noted. On the other side, heights corresponding to interdigitated regions were not found for the sample prepared at 5000 rpm.

These results indicate that at higher rotation speeds the fraction of interdigitated regions decreases. We speculate that higher rotation speeds could facilitate the layer rupture reducing the lateral tension of the bilayer and hence decreasing the fraction of the interdigitate phase $\mathrm{L}_{\beta} l$. Therefore, the interdigitated area would be affected by the rotation speed used to prepare the sample and it could be reduced or increased by adjusting this parameter properly.

\subsection{Effect of cholesterol}

Cholesterol is known to affect interdigitation in hydrated samples [38, 40-42] and also to affect the lateral mobility of lipid layers [54-57]. The addition of Cholesterol favours the presence of the liquidordered phase, $L_{0}$, which has intermediate properties between $L_{\beta}$ and $L_{\alpha}$. 


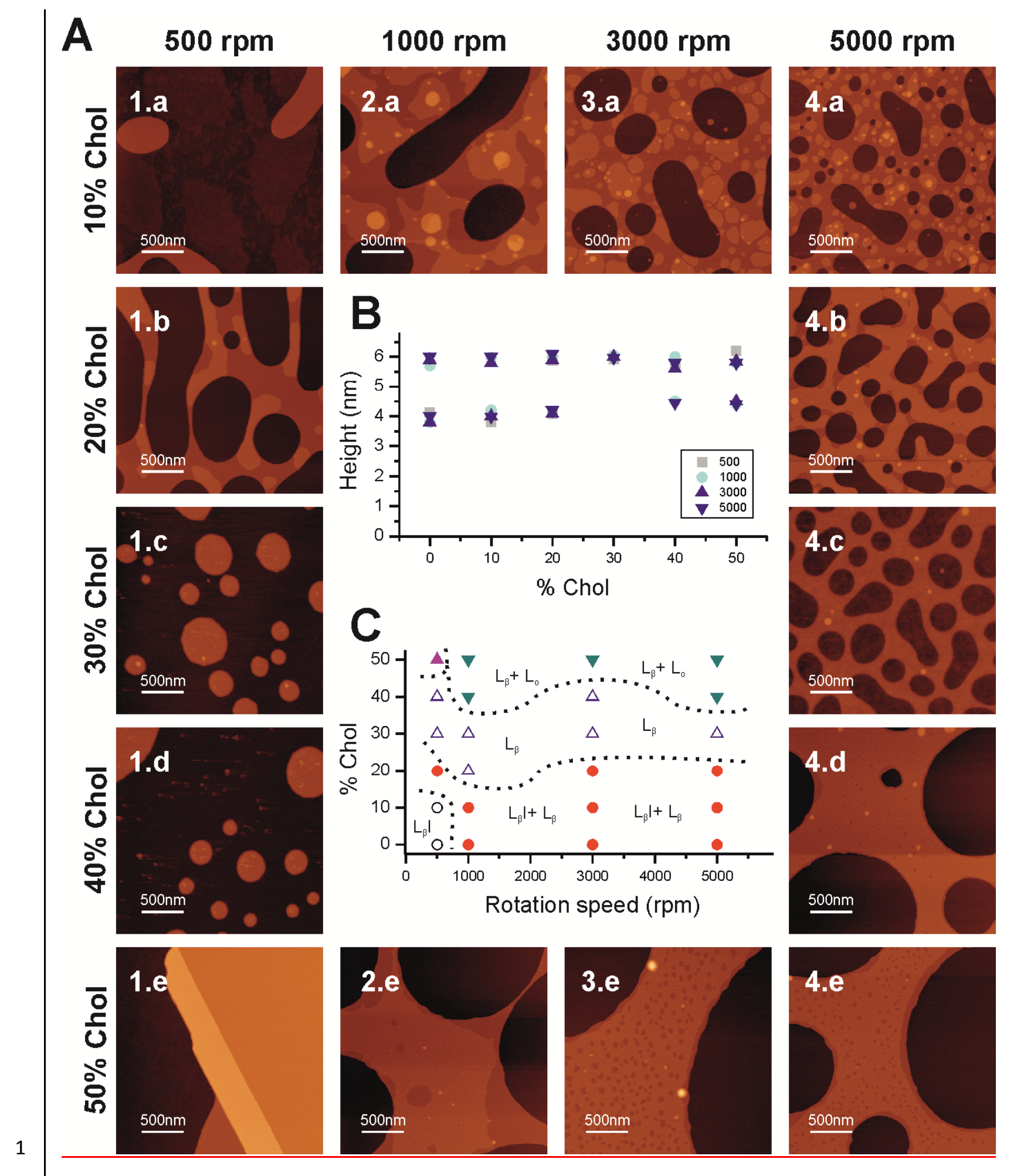

2 Figure 5. A) AFM topography images of spin-coated samples prepared at different rotation speeds, 500 $3 \mathrm{rpm}(1), 1000 \mathrm{rpm}$ (2), $3000 \mathrm{rpm}$ (3) and $5000 \mathrm{rpm}$ (4); and containing 10\% (a), 20\% (b), 30\% (c), 40\%(d) 4 and $50 \%$ (e) of Chol. Z-scale $=30 \mathrm{~nm}$. B) Plot representation of the bilayer height extracted from the 
topography images vs $\%$ Chol. Grey squares $=500 \mathrm{rpm}$, clear blue dots $=1000 \mathrm{rpm}$, dark blue triangle $=$ $3000 \mathrm{rpm}$ and inverted blue triangles $=5000 \mathrm{rpm}$. C) phase representation interpreted from height analysis. Empty dots $=L_{\beta} l$, red dots $=$ coexistence of $L_{\beta} l$ and $L_{\beta}$, empty triangles $=L_{\beta}$, inverted full triangles $=$ coexistence of $L_{\beta}$ and $L_{o}$ and purple triangles $=$ Chol crystals and DPPC layers.

The influence of Chol at different rotation speeds on the interdigitation in dried samples was tested for DPPC, chosen as model PC for this purpose. Figure 5 shows AFM images of dry DPPC lipid layers prepared with Chol concentrations ranging from $10 \%$ to $50 \%$ and prepared at rotation speeds from 500 $\mathrm{rpm}$ to $5000 \mathrm{rpm}$. The characteristic thicknesses of the lipid layers present in the samples are shown in Fig. 5B as a function of the Chol concentrations for the different rotation speeds.

Samples with 0-10\% Chol (Fig.4, Fig. 5A a, Fig. 5B and 5C) presented coexistence of two heights associated to interdigitated and non-interdigitated regions, except samples prepared at $500 \mathrm{rpm}$ (Fig.4 and Fig.5A.1a) in which only heights around $4 \mathrm{~nm}$ (assigned to interdigitated regions) were observed. At $20 \%$ Chol samples (Fig. 5A b, Fig 5B, Fig 5C) presented again coexistence, except the sample prepared at $1000 \mathrm{rpm}$ in which only $L_{\beta}$ was represented (image not shown, Fig. $5 B-C$ ). At $30 \%$ of Chol all samples presented heights in accordance with $L_{\beta}$ independently of the rotation speed used, without observable presence of interdigitated phase or liquid ordered phase (Fig.5A c, Fig. 5B-C). Above $30 \%$ some samples presented heights corresponding to $L_{\beta}$ in coexistence with lower regions (Fig.5B). Except in samples prepared with $50 \%$ Chol at $500 \mathrm{rpm}$ (Fig.5A.1e), in which the presence of Chol crystals and bilayers with thickness in accordance with $L_{\beta}$ were observed. It is known that above $50 \%$ Chol DPPC becomes saturated and Chol precipitates, this observation is in accordance with previous results. [58]

$\mathrm{L}_{\beta}$ phase is easily distinguishable, while discriminating between interdigitated or liquid ordered phase is difficult as both phases present characteristics heights of $\sim 4 \mathrm{~nm}[28,43]$. However, we could observe a clear change in the shape, distribution and size of the thinner regions with the increment of the cholesterol ratio (Figure 5.A). At low Chol contents the predominant phase would be interdigitated due to the small presence of $\mathrm{Chol}$ and the presence of lateral tensions. On the other hand, the lower regions are not observable in $30 \%$ Chol content. Our interpretation is the following: the incorporation of Chol to the DPPC helps to the fluidization of the lipid layer and to its lateral mobility. The absence of lower regions at $30 \%$ of Chol could indicate a threshold value in which the lipid layer has enough fluidity to avoid the interdigitation. For values above that, the layer is not only affected by the fluidity, and the 
presence of liquid ordered regions is more evident. Thus, at low amounts of Chol lower regions would correspond, mostly, to interdigitated regions, while above $30 \%$ they could be mostly associated to liquid ordered regions (Figure 5.C). This hypothesis not only takes into account the change in the shape and distribution of the domains, but also the change in the shape of the lipid layers which showed patterns in accordance with an increased fluidity [22].

These results indicate that Chol can modulate the presence of interdigitated regions in DPPC samples prepared by spin-coating in air. Moreover, the addition of a certain quantity (in here $30 \% \mathrm{Chol}$ ) allows obtaining homogeneous heights and avoid the effect provoked by the lateral tension.

\section{Conclusions}

In the present study we have analyzed the presence of lower regions associated to interdigitated phase ultrathin (single layer) spin-coated saturated lipid layers supported on mica in dry air. We have observed that lipids with longer acyl chains, such as DPPC and DSPC, show the presence of bilayers with an intermediate thickness between a bilayer and a monolayer, which is in accordance with the height associated to interdigitated phase. Instead, low temperature transition temperature lipid samples, such as DLPC and DMPC, do not show lower regions. We showed that the presence of alcohols do not affect the total area of the interdigitated area (contrary to what happens in hydrated samples) while the lateral pressure (represented by the spin-coating rotation speed) and cholesterol content have a remarkable influence on their presence. In fact, the lateral tension due to the rotation speed was demonstrated to be at the origin of interdigitation in dried bilayers prepared by spin-coating. We showed that by adjusting the preparation procedure or the composition, for instance by adding Cholesterol at certain concentrations, interdigitation can be reduced drastically or even suppressed, and flat homogeneous samples can be obtained. This shows that, in addition to the case of unsaturated lipids, it is also possible to prepare high quality planar lipid bilayer samples stable in dry environment with saturated lipids.

\section{ACKNOWLEDGMENT}


1 This work was partially supported by the Spanish MEC under grant TEC2016-79156-P. G.G.

2 acknowledges support from the ICREA Academia program from the Generalitat de Catalunya. A.D.-P.

3 thanks M. Cazorla from the Nanotechnology Platform of IBEC for her assistance during the spin-coating depositions.

5

\section{References}

[1] A.A. Brian, H.M. McConnell, Allogeneic stimulation of cytotoxic T cells by supported planar membranes., Proceedings of the National Academy of Sciences of the United States of America 81 (1984) 6159-6163.

[2] E. Sackmann, Supported membranes: scientific and practical applications., Science (New York, N.Y.) 271 (1996) 43-48.

[3] M. Edidin, The state of lipid rafts: from model membranes to cells., Annual review of biophysics and biomolecular structure 32 (2003) 257-83.

[4] K. Simons, W.L.C. Vaz, Model systems, lipid rafts, and cell membranes., Annual review of biophysics and biomolecular structure 33 (2004) 269-95.

[5] K. Jacobson, O. Mouritsen, R. Anderson, Lipid rafts: at a crossroad between cell biology and physics, Nature cell biology 9 (2007) 7-14.

[6] Y.-H.M. Chan, S.G. Boxer, Model membrane systems and their applications, Current Opinion in Chemical Biology 11(6) (2007) 581-587.

[7] K. El Kirat, S. Morandat, Y.F. Dufrêne, Nanoscale analysis of supported lipid bilayers using atomic force microscopy., Biochimica et biophysica acta 1798 (2010) 750-65.

[8] S.G. Boxer, Molecular transport and organization in supported lipid membranes, Current Opinion in Chemical Biology 4(6) (2000) 704-709.

[9] E. Castellana, P. Cremer, Solid supported lipid bilayers: From biophysical studies to sensor design, Surface Science Reports 61 (2006) 429-444.

[10] E. Reimhult, K. Kumar, Membrane biosensor platforms using nano- and microporous supports, Trends in Biotechnology 26(2) (2008) 82-89. 
[11] S.S. Hinman, Q. Cheng, Bioinspired assemblies and plasmonic interfaces for electrochemical biosensing, Journal of Electroanalytical Chemistry 781 (2016) 136-146.

[12] A.C. Simonsen, L.A. Bagatolli, Structure of spin-coated lipid films and domain formation in supported membranes formed by hydration., Langmuir : the ACS journal of surfaces and colloids 20 (2004) 9720-9728.

[13] E. Ten Grotenhuis, W.J.M. Van Der Kemp, J.G. Blok, J.C. Van Miltenburg, J.P. Van Der Eerden, Scanning force microscopy of cholesterol multilayers prepared with the spin-coating technique, Colloids and Surfaces B: Biointerfaces 6 (1996) 209-218.

[14] J. Generosi, C. Castellano, D. Pozzi, A.C. Castellano, R. Felici, F. Natali, G. Fragneto, X-ray and neutron reflectivity study of solid-supported lipid membranes prepared by spin coating, Journal of Applied Physics 96 (2004) 6839.

[15] A.C. Simonsen, Activation of phospholipase A2 by ternary model membranes., Biophysical journal 94 (2008) 3966-3975.

[16] U. Mennicke, T. Salditt, Preparation of Solid-Supported Lipid Bilayers by Spin-Coating, Langmuir 18 (2002) 8172-8177.

[17] M.H. Jensen, E.J. Morris, A.C. Simonsen, Domain shapes, coarsening, and random patterns in ternary membranes, Langmuir 23 (2007) 8135-8141.

[18] G. Pompeo, M. Girasole, a. Cricenti, F. Cattaruzza, a. Flamini, T. Prosperi, J. Generosi, a.C. Castellano, AFM characterization of solid-supported lipid multilayers prepared by spin-coating., Biochimica et biophysica acta 1712 (2005) 29-36.

[19] M. Jurak, E. Chibowski, Wettability and topography of phospholipid DPPC multilayers deposited by spin-coating on glass, silicon, and mica slides., Langmuir : the ACS journal of surfaces and colloids 23 (2007) 10156-10163.

[20] L. Krapf, M. Dezi, W. Reichstein, J. Köhler, S. Oellerich, AFM characterization of spin-coated multilayered dry lipid films prepared from aqueous vesicle suspensions, Colloids and Surfaces B: Biointerfaces 82 (2011) 25-32.

[21] A. Dols-Perez, L. Fumagalli, A.C. Simonsen, G. Gomila, Ultrathin spin-coated dioleoylphosphatidylcholine lipid layers in dry conditions: A combined atomic force microscopy and nanomechanical study, Langmuir 27 (2011) 13165-13172. 
4

[22] A. Dols-Perez, L. Fumagalli, G. Gomila, Structural and nanomechanical effects of cholesterol in binary and ternary spin-coated single lipid bilayers in dry conditions, Colloids and Surfaces B:

Biointerfaces 116 (2014) 295-302.

[23] E. Reimhult, F. Höök, B. Kasemo, Intact vesicle adsorption and supported biomembrane formation from vesicles in solution: Influence of surface chemistry, vesicle size, temperature, and osmotic pressure, Langmuir 19 (2003) 1681-1691.

[24] L.K. Tamm, H.M. McConnell, Supported phospholipid bilayers., Biophysical journal 47 (1985) 105113.

[25] S.-Y. Jung, M.a. Holden, P.S. Cremer, C.P. Collier, Two-Component Membrane Lithography via Lipid Backfilling, ChemPhysChem 6 (2005) 423-426.

[26] S. Lenhert, C.a. Mirkin, H. Fuchs, In situ lipid dip-pen nanolithography under water., Scanning 32 (2010) 15-23.

[27] U. Vierl, L. Löbbecke, N. Nagel, G. Cevc, Solute effects on the colloidal and phase behavior of lipid bilayer membranes: ethanol-dipalmitoylphosphatidylcholine mixtures., Biophysical journal 67 (1994) 1067-1079.

[28] J. Mou, J. Yang, C. Huang, Z. Shao, Alcohol induces interdigitated domains in unilamellar phosphatidylcholine bilayers., Biochemistry 33 (1994) 9981-9985.

[29] S.A. Simon, T.J. McIntosh, Interdigitated hydrocarbon chain packing causes the biphasic transition behavior in lipid/alcohol suspensions, BBA - Biomembranes 773(1) (1984) 169-172.

[30] J.A. Barry, K. Gawrisch, Direct NMR evidence for ethanol binding to the lipid-water interface of phospholipid bilayers, Biochemistry 33(26) (1994) 8082-8088.

[31] T. Adachi, H. Takahashi, K. Ohki, I. Hatta, Interdigitated structure of phospholipid-alcohol systems studied by x-ray diffraction., Biophysical journal 68 (1995) 1850-1855.

[32] J.A. Barry, K. Gawrisch, Effects of ethanol on lipid bilayers containing cholesterol, gangliosides, and sphingomyelin, Biochemistry 34(27) (1995) 8852-8860.

[33] L.L. Holte, K. Gawrisch, Determining ethanol distribution in phospholipid multilayers with MASNOESY spectra, Biochemistry 36(15) (1997) 4669-4674. 
[34] K.J. Tierney, D.E. Block, M.L. Longo, Elasticity and phase behavior of DPPC membrane modulated by cholesterol, ergosterol, and ethanol., Biophysical journal 89 (2005) 2481-2493.

[35] J.T. Marquês, A.S. Viana, R.F.M. De Almeida, Ethanol effects on binary and ternary supported lipid bilayers with gel/fluid domains and lipid rafts, Biochimica et Biophysica Acta (BBA) - Biomembranes 1808(1) (2011) 405-414.

[36] T. Hata, H. Matsuki, S. Kaneshina, Effect of local anesthetics on the bilayer membrane of dipalmitoylphosphatidylcholine: interdigitation of lipid bilayer and vesicle-micelle transition, Biophysical Chemistry 87(1) (2000) 25-36.

[37] K. Takeda, H. Okuno, T. Hata, M. Nishimoto, H. Matsuki, S. Kaneshina, Interdigitation and vesicle-tomicelle transformation induced by a local anesthetic tetracaine in phospholipids bilayers, Colloids and Surfaces B: Biointerfaces 72(1) (2009) 135-140.

[38] J.M. Vanegas, R. Faller, M.L. Longo, Influence of ethanol on lipid/sterol membranes: Phase diagram construction from AFM imaging, Langmuir 26 (2010) 10415-10418.

[39] C. Xing, O.H.S. Ollila, I. Vattulainen, R. Faller, Asymmetric nature of lateral pressure profiles in supported lipid membranes and its implications for membrane protein functions, Soft Matter 5 (2009) 3258-3261.

[40] H. Komatsu, Effect of cholesterol on the ethanol-induced interdigitated gel phase in phosphatidylcholine: Use of fluorophore pyrene-labeled phosphatidylcholine, Biochemistry 30(9) (1991) 2463-2470.

[41] M.F.N. Rosser, H.M. Lu, P. Dea, Effects of alcohols on lipid bilayers with and without cholesterol: The dipalmitoylphosphatidylcholine system, Biophysical Chemistry 81(1) (1999) 33-44.

[42] J.M. Vanegas, M.F. Contreras, R. Faller, M.L. Longo, Role of unsaturated lipid and ergosterol in ethanol tolerance of model yeast biomembranes, Biophysical Journal 102(3) (2012) 507-516.

[43] L. Redondo-Morata, M.I. Giannotti, F. Sanz, Influence of cholesterol on the phase transition of lipid bilayers: A temperature-controlled force spectroscopy study, Langmuir 28 (2012) 12851-12860.

[44] A. Alessandrini, P. Facci, Phase transitions in supported lipid bilayers studied by AFM, Soft Matter 10(37) (2014) 7145-7164. 
[45] I. Horcas, R. Fernández, J.M. Gómez-Rodríguez, J. Colchero, J. Gómez-Herrero, a.M. Baro, WSXM: a software for scanning probe microscopy and a tool for nanotechnology., The Review of scientific instruments 78 (2007) 013705.

[46] L. Perino-Gallice, G. Fragneto, U. Mennicke, T. Salditt, F. Rieutord, Dewetting of solid-supported multilamellar lipid layers, European Physical Journal E 8 (2002) 275-282.

[47] F. Tokumasu, A.J. Jin, G.W. Feigenson, J.a. Dvorak, Nanoscopic lipid domain dynamics revealed by atomic force microscopy., Biophysical journal 84 (2003) 2609-18.

[48] S. Garciamanyes, G. Oncins, F. Sanz, Effect of pH and ionic strength on phospholipid nanomechanics and on deposition process onto hydrophilic surfaces measured by AFM, Electrochimica Acta 51 (2006) 5029-5036.

[49] F. Yarrow, B.W.M. Kuipers, AFM study of the thermotropic behaviour of supported DPPC bilayers with and without the model peptide WALP23, Chemistry and Physics of Lipids 164(1) (2011) 9-15.

[50] B.a. Lewis, D.M. Engelman, Lipid bilayer thickness varies linearly with acyl chain length in fluid phosphatidylcholine vesicles., Journal of molecular biology 166 (1983) 211-217.

[51] L. Toppozini, C.L. Armstrong, M.A. Barrett, S. Zheng, L. Luo, H. Nanda, V.G. Sakai, M.C. Rheinstadter, Partitioning of ethanol into lipid membranes and its effect on fluidity and permeability as seen by X-ray and neutron scattering, Soft Matter 8(47) (2012) 11839-11849.

[52] A.C. Simonsen, Spatiotemporal Organization of Spin-Coated Supported Model Membranes, in: R. Faller, M.L. Longo, S.H. Risbud, T. Jue (Eds.), Biomembrane Frontiers: Nanostructures, Models, and the Design of Life, Humana Press, Totowa, NJ, 2009, pp. 141-170.

[53] M. Gedig, S. Faiß, A. Janshoffa, Melting and interdigitation of microstructured solid supported membranes quantified by imaging ellipsometry, Biointerphases 3(2) (2008) FA51-FA58.

[54] T. Parasassi, a.M. Giusti, M. Raimondi, E. Gratton, Abrupt modifications of phospholipid bilayer properties at critical cholesterol concentrations., Biophysical journal 68 (1995) 1895-1902.

[55] S. Raffy, J. Teissie, Control of lipid membrane stability by cholesterol content, Biophysical Journal 76 (1999) 2072-2080.

[56] D. Marsh, Liquid-ordered phases induced by cholesterol: A compendium of binary phase diagrams, Biochimica et Biophysica Acta - Biomembranes 1798 (2010) 688-699. 
1 [57] F. de Meyer, B. Smit, Effect of cholesterol on the structure of a phospholipid bilayer., Proceedings of 2 the National Academy of Sciences of the United States of America 106 (2009) 3654-3658.

3

4

5

6

7

\section{AUTHOR INFORMATION}

\section{$9 \quad$ Author Contributions}

10

The manuscript was written through contributions of all authors. ADP prepared the samples,

11

12

[58] R.M. Epand, D. Bach, E. Wachtel, In vitro determination of the solubility limit of cholesterol in phospholipid bilayers, Chemistry and Physics of Lipids 199 (2016) 3-10. characterized them and analysed the results. LF and GG coordinated the investigation. All authors wrote the manuscript and approved the final version. 
2 GRAPHICAL ABSTRACT

3
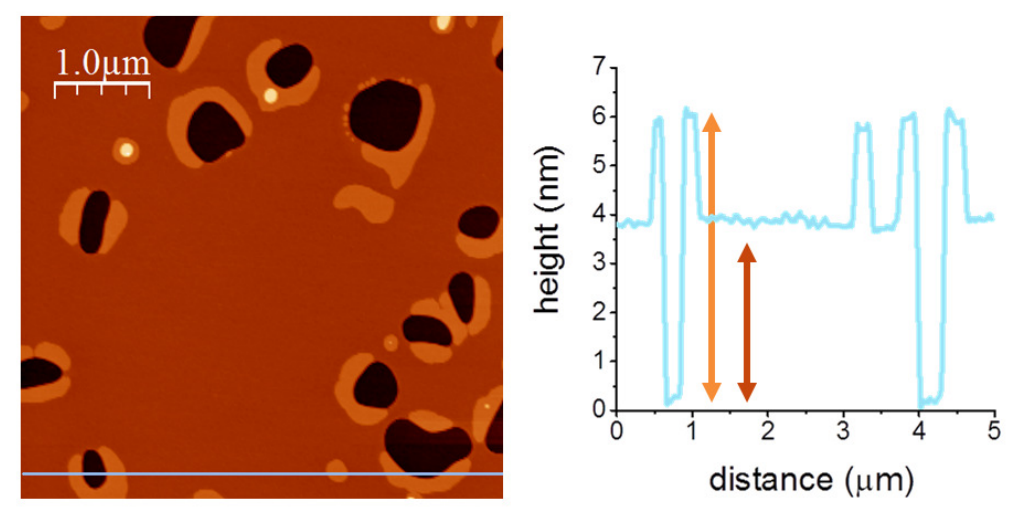